\title{
Fully integrated SDM amplifiers
}

\author{
Yongmin Jung, Saurabh Jain, Shaif-Ul Alam and David J. Richardson \\ Optoelectronics Research Centre, University of Southampton, Southampton, SO17 1BJ, UK \\ (Invited paper)
}

\begin{abstract}
Recent research advances in Space-Division-Multiplexing (SDM) technology will be reviewed with a particular focus on the development of passive optical components and amplifiers. In particular, 32-core multicore fiber isolators and amplifiers are discussed in detail.
\end{abstract}

\section{INTRODUCTION}

Multiple spatial channels in an optical fiber can be used as independent data streams in optical transmission, which is called as "Space Division Multiplexing (SDM)" [1-2], and various types of SDM fibers such as few mode fibers and multicore fibers have been investigated to increase the data capacity of the current fiber optic transmission system based on single mode fibers. A wide range of new SDM components and SDM amplifiers have accordingly been developed and recently received much attention due to their potential cost, energy and space saving effects. In this paper, we will overview the recent progress on the SDM components and SDM amplifiers including the state-of-the-art 32-core multicore fiber (MCF) isolators [3] and 32-core MCF amplifiers [4]. Additionally, these independent multiple spatial amplifier channels provided by MCF offer attractive opportunities for new types of laser supporting multiple laser output or multi-wavelengths and an exemplary 7wavelengths fiber laser based on MCF technology [5] will be presented.

\section{32-CORE MCF ISOLATOR}

Compact micro-optic collimator assembly is the one of the key optical platform for fiber optic components and we have recently integrated SDM fibers with this fiberto-fiber coupling scheme. Bulk optic component can be simply plugged into the beam path whilst providing low insertion loss and preserving the integrity of the spatial channels offering significant scope for cost reduction (relative to the use of an array of single mode devices offering a similar total number of spatial channels). Figure 1(a) shows a schematic diagram of a 32-core MCF collimator assembly [3] consisting of a micro-optic lens and a MCF ferrule, fitted in a ferrule sleeve. Compact fiber optic collimators usually use GRIN lens or C-lens

This work was supported by the EU-Japan coordinated R\&D project on "Scalable And Flexible optical Architecture for Reconfigurable Infrastructure (SAFARI)" by the Ministry of Internal Affairs and Communications (MIC) of Japan and EC Horizon 2020 and by the EPSRC funded "AirGuide Photonics" Programme Grant (EP/P030181/1). elements to transform the emergent light from an input 32-core MCF into a collimated free-space beam that can then be refocused into another MCF using a second identical assembly in reverse. Note that the MCF collimators intrinsically require rotational alignment and the far field intensity distribution from the collimator was examined using a visible $\mathrm{He}-\mathrm{Ne}$ laser for rough angular alignment before fine adjustment with an optical power meter. As shown in Fig. 1(b) the average insertion loss was less than $1.5 \mathrm{~dB}$ and the core-to-core variation was less than $1.5 \mathrm{~dB}$. Optical isolation was more than $32 \mathrm{~dB}$ over the full C-band the inter-core crosstalk was less than $-40 \mathrm{~dB}$. Note that the micro-optic collimator technology can be applicable to other types of the SDM fibers and few mode fiber isolators [6] have demonstrated with low insertion loss $(<1 \mathrm{~dB})$.

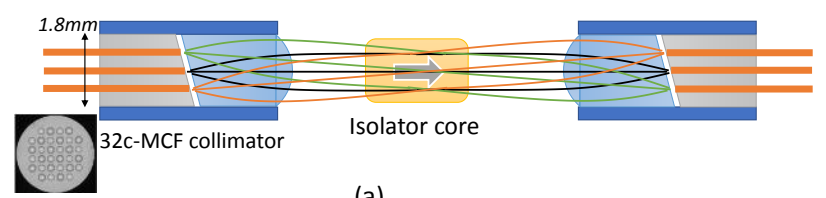

(a)

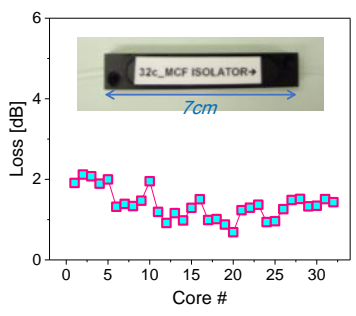

\begin{tabular}{|c|c|}
\hline Optical Parameters & Typical \\
\hline Number of cores & 32 cores \\
\hline Wavelength range & Full C-band \\
\hline Average insertion loss & $<1.5 \mathrm{~dB}$ \\
\hline Core-to-core IL variation & $<1.5 \mathrm{~dB}$ \\
\hline Optical isolation & $>32 \mathrm{~dB}$ \\
\hline Back reflection & $<-32 \mathrm{~dB}$ \\
\hline
\end{tabular}

(b)

Fig. 1. (a) Schematic of fully integrated 32-core MCF isolator and (b) their optical characteristics.

\section{32-CORE MCF AMPLIFIER}

Figure 2 shows the schematic setup for a 32-core MCF amplifier [4] in a cladding-pumped configuration. A $7 \mathrm{~m}$ of erbium/ytterbium doped 32-core MCF was used as a gain medium and a side coupler scheme was employed to couple the multimode pump light into the active fiber and more than $60 \%$ pump coupling efficiency can be readily achieved. Passive fiber was directly spliced to an active fiber and the fiber splicer loss was estimated to be about $1.3 \mathrm{~dB}$ due to the mode field diameter mismatch between two fibers. Note that the fully integrated 32-core isolators are placed at both input and output end of the amplifier to suppress parasitic lasing and any unwanted feedback into the amplifier. The bottom of Fig. 2 shows the gain performance of the 32-core MCF amplifier for $-4 \mathrm{dBm}$ 
input signal power. A minimum gain of $>17 \mathrm{~dB}$ and an averaged noise figure of $6.5 \mathrm{~dB}$ was measured over all cores in the wavelength range $1534-1561 \mathrm{~nm}$. The coreto-core variation for both amplifier gain and NF was measured to be less than $2 \mathrm{~dB}$. This amplifier was also tested in a MCF loop system and transmission over distances $>1850 \mathrm{~km}$ was successfully demonstrated for 100Gbit/s QPSK signal [4].
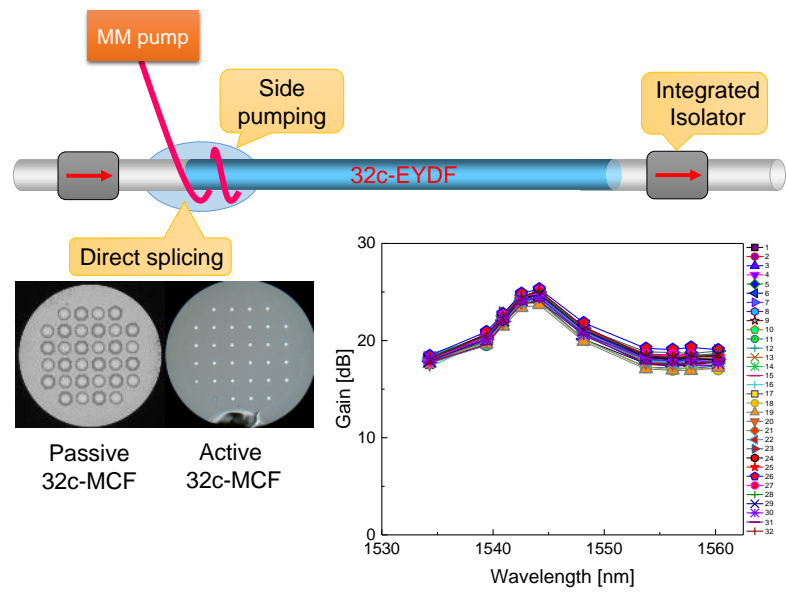

Fig. 2. Schematic (top) and gain performance (bottom) of the 32-core MCF amplifier.

\section{Multi-WAVELENGTH FIBER LASER}

Each core of the multicore erbium doped fiber can be used as an independent gain medium for fiber lasers and an exemplary multi-wavelength fiber laser [5] was constructed using a 7-core EDF. As shown in Fig. 3(a), a $6 \mathrm{~m}$ long, 7-core EDF was used as an independent optical gain fiber and a linear laser cavity was constructed with a Sagnac loop mirror on the left and 4\% Fresnel reflection from a flat fiber end facet on the right. As a wavelength selective element, an arrayed waveguide grating (AWG) was used to determine the lasing wavelength of the individual cores in the laser cavity and a Sagnac loop filter to provide a much sharper passband for each channel. Two laser output ports are available in this laser configuration; multi-wavelength laser output from a single mode fiber (output-1) and single-wavelength multiple core beam from the MCF (output-2). Fig. 3(b) shows a typical optical spectrum of the multi-wavelength laser output (output-1) and seven distinct lasing wavelengths are clearly obtained with a wavelength spacing of $1.6 \mathrm{~nm}$. The $3 \mathrm{~dB}$ bandwidth of each wavelength is measured to be less than $0.02 \mathrm{~nm}$ and $20 \mathrm{~dB}$ bandwidth of the laser is $\sim 0.07 \mathrm{~nm}$. Importantly, the lasing wavelengths of the multi-wavelength fiber laser are determined by the choice of the output ports of the AWG module and any combination of wavelengths can be generated on a $100 \mathrm{GHz}$ grid. At the free space output port (output-2), multiple laser beams are emitted from the individual core of the MCF and have different lasing wavelengths and propagate in different angular directions. Such multi-wavelength fiber lasers may find application in WDM communications, fiber optic sensors,
LIDAR, microwave generation and high resolution spectroscopy.

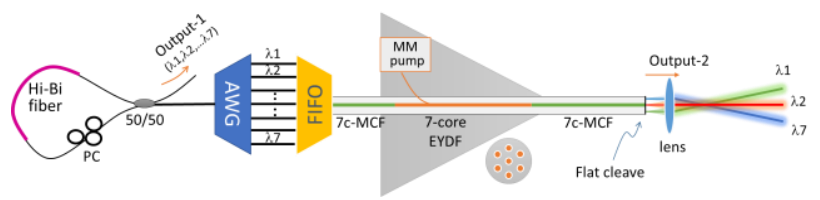

(a)

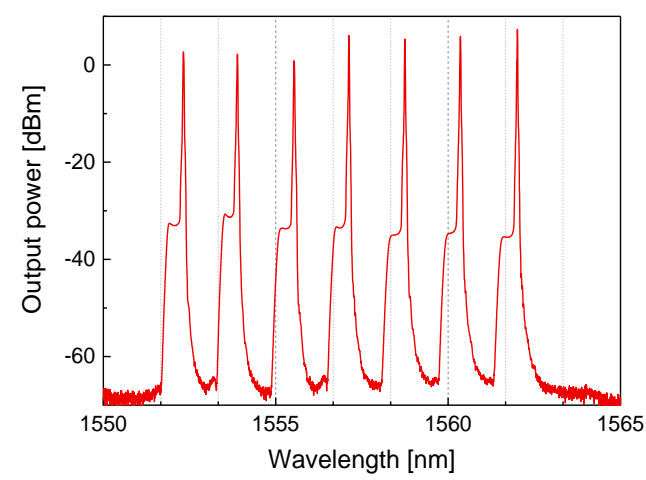

(b)

Fig. 3. (a) Schematic of the multi-wavelength fiber laser and (b) their typical optical spectrum.

\section{CONClusions}

Over the last few years, SDM amplifiers have been integrated in a fully fiberized format by employing inline passive SDM components. Current state-of-the-art SDM amplifier, a 32-core multicore fiber amplifier, has been fully fiberized in a cladding pumped configuration and a good amplifier performance and significant component sharing has been successfully demonstrated. We believe that it is a great improvement in SDM amplifier in terms of integration but it is also anticipated that these SDM amplifier technology can be applicable to new types of fiber laser development having multiple output beams or multi-wavelengths.

\section{REFERENCES}

[1] D. J. Richardson et al., "Space-division multiplexing in optical fibres," Nature Photonics 7, pp. 354-362, 2013.

[2] V.A.J.M. Sleiffer et al., "Field demonstration of modedivision multiplexing upgrade scenarios on commercial networks," Opt. Express 21, pp. 31036-31046, 2013.

[3] Y. Jung et al., "Compact 32-core multicore fibre isolator for high-density spatial division multiplexed transmission," ECOC'16, paper W2.B4, Dusseldorf, Germany, 18-22 September 2016.

[4] S. Jain et al., "32-core erbium/ytterbium-doped multicore fiber amplifier for next generation space-division multiplexed transmission system," Opt. Express 25, pp. 32887-32896, 2017.

[5] Y. Jung et al., "Multi-wavelength fiber laser using a single multicore erbium doped fiber," OFC'18, paper M2J.6, San Diego, CA, USA, 3-7 March 2018.

[6] Y. Jung et al., "Compact few-mode fiber collimator and associated optical components for mode division multiplexed transmission," OFC'16, Paper W2A. 40, Anaheim, USA, 20-24 March 2016. 